\title{
Usual gait speed is associated with frailty status, institutionalization, and mortality in community- dwelling rural older adults: a longitudinal analysis of the Aging Study of Pyeongchang Rural Area
}

This article was published in the following Dove Press journal:

Clinical Interventions in Aging

\author{
Hee-Won Jung ${ }^{1,2, *}$ \\ II-Young Jang ${ }^{3,4, *}$ \\ Chang Ki Lee ${ }^{5}$ \\ Sang Soo $\mathrm{Yu}^{4}$ \\ Jae Kyoon Hwang ${ }^{4}$ \\ Chawoong Jeon ${ }^{4}$ \\ Young Soo Lee $^{3}$ \\ Eunju Lee ${ }^{3}$
}

'Graduate School of Medical Science and Engineering, Korea Advanced Institute of Science and Technology, Daejeon, Republic of Korea; ${ }^{2}$ Geriatric Center, Seoul National University Bundang Hospital, Seongnam-si, Gyeonggi-do, Republic of Korea; ${ }^{3}$ Department of Internal Medicine, Asan Medical Center, University of Ulsan College of Medicine, Seoul, Republic of Korea; ${ }^{4}$ PyeongChang Health Center \& County Hospital, PyeongChang-gun, Gangwon-Do, Republic of Korea; ${ }^{5}$ Goldman Urology Clinic, Seoul, Republic of Korea

*These authors contributed equally to this work

\begin{abstract}
Purpose: We aimed to describe the age- and sex-specific distributions of gait speed and to evaluate associations with longitudinal outcomes in Korean rural community-dwelling older adults.
\end{abstract}

Patients and methods: A total of 1,348 people (mean age: 76 years, 55\% women) in the population-based, prospective cohort of Aging Study of Pyeongchang Rural Area (ASPRA) between October 2014 and June 2017. All participants underwent a comprehensive geriatric assessment, including 4-m usual gait speed, and were followed annually.

Results: Among the 1,348 participants, women had a slower gait speed than men (mean $0.709 \mathrm{~m} / \mathrm{s}$ vs $0.850 \mathrm{~m} / \mathrm{s}, P<0.001$ ). Gait speed was inversely associated with age, frailty index; slow gait speed as classified by sex-specific quartile was associated with the prevalence of common geriatric syndromes. During the mean follow-up period of 21.5 months (SD 7.88), future survival without mortality or institutionalization was affected by sex-specific gait-speed quartile ( $\log$ rank test $P<0.001)$ : the 1 st quartile of sex-specific gait speed was associated with increased risk of death or institutionalization.

Conclusion: Gait speed was related to age, sex, frailty status, and geriatric health outcomes in Korean rural community-dwelling older adults. Since this gait-speed distribution in an older Korean population differs from previous data on other populations, we should consider a gaitspeed cutoff value based on sex-specific quartiles to prevent misclassification in sarcopenia and frailty diagnosis.

Keywords: cohort studies, frailty, gait speed, geriatric assessment, physical function

\section{Introduction}

Gait speed in older adults has been accepted as a reliable and sensitive measure for assessment of physiologic performance and prediction of clinical outcomes, and indeed has been regarded as the "sixth vital sign." ", A ground-breaking study ${ }^{3}$ on nine Western cohorts showed that gait speed can predict survival in older people as precisely as age, sex, and use of mobility aids. Given its clinical, epidemiological importance, gait speed has been included in most clinical studies dealing with older adults.

Frailty is defined as a state of decreased physiological reserve coupled with increased vulnerability to possible stressors in older adults. ${ }^{4}$ Cumulative research results have demonstrated its outstanding performance in predicting clinical geriatric outcomes under various settings. In defining physical frailty, objective gait speed ${ }^{5}$ or subjective walking capability ${ }^{6}$ usually has been included in operational frailty criteria.
Department of Internal Medicine, Asan

Medical Center, University of Ulsan

College of Medicine, 88, Olympic-ro

43-gil, Songpa-gu, Seoul, 05505,

Republic of Korea

$\mathrm{Tel}+82230103308$

Fax +82 24760824

Email eunjulee@amc.seoul.kr

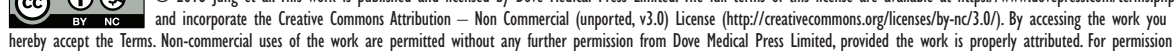
for commercial use of this work, please see paragraphs 4.2 and 5 of our Terms (https://www.dovepress.com/terms.php). 
Moreover, studies have measured gait speed as the sole marker of physiological reserve and have shown that it can predict adverse procedural outcomes. ${ }^{7,8}$

Gait speed, accordingly, has been included as the component of diagnostic criteria for sarcopenia. ${ }^{9}{ }^{10}$ A recent definition of sarcopenia uses physical performance, muscle strength, and muscle mass as indicators. A report of the European Working Group on Sarcopenia, having summarized several studies from Western countries, suggested a $0.8 \mathrm{~m} / \mathrm{s}$ gait-speed cutoff value for detection of sarcopenia. ${ }^{5,11,12}$ The Asian Working Group for Sarcopenia (AGWS), notwithstanding the possible gender differences and large heterogeneities between studies from various countries, also suggested the $0.8 \mathrm{~m} / \mathrm{s}$ cutoff value of gait speed to detect sarcopenia. ${ }^{10}$

South Korea is one of the fastest aging societies in the world, and the estimated life expectancy of Korean people will be the longest by 2,030 among developed countries. ${ }^{13}$ Because of this compelling need for research on frailty and sarcopenia, studies thus far have revealed the prevalence and clinical implications of frailty ${ }^{14}$ and sarcopenia ${ }^{15,16}$ in Korean populations. However, to date, no study has determined the gait-speed distribution or analyzed its clinical implications in older Korean adults. Consequently, most studies on frailty and sarcopenia in Korea have had to adopt a gait-speed cutoff value for a Western population, restricting scientific and clinical validity of their results in the Korean population.

In this context, we aimed to determine the age- and sexspecific distributions of usual gait speed and to analyze the longitudinal associations of usual gait speed with outcomes of mortality and institutionalization in a rural communitybased prospective cohort study designed to reflect the general Korean rural population.

\section{Patients and methods \\ Study design and population}

The current study population was selected from the Aging Study of PyeongChang Rural Area (ASPRA), a populationbased, prospective cohort study on frailty and geriatric syndromes among older Koreans living in rural areas. ASPRA's design and the method by which it was carried out are described elsewhere. ${ }^{15}$ Briefly, participants residing in Pyeongchang County, Gangwon Province, Korea, located $180 \mathrm{~km}$ east of Seoul, were enrolled. The inclusion criteria for participation were 1) aged $\geq 65$ years; 2) registered in the National Healthcare Service; 3) ambulatory with or without an assistive device; 4) living at home; and 5) able to provide informed consent. We excluded those who were
1) living in a nursing home; 2) hospitalized; or 3) bed-ridden and receiving nursing home-level care at home at the time of enrollment.

In the present study, we used data from ASPRA's 1,350participant cohort recruited and baseline assessed between October 2014 and June 2017. This included over 90\% of eligible older adults living in the study area. A previous report showed that, based on a nationally representative sample of the Korea National Health and Nutrition Examination Survey (KNHANES), the characteristics of the ASPRA participants were very similar to the general Korean rural population, except for a higher proportion of agricultural laborers and a low formal-education level. ${ }^{15}$ All of the participants underwent a comprehensive geriatric assessment every 12 months. Participants who died or were institutionalized for $>3$ months were recorded as health-related outcomes. The protocol of this study was approved by the Institutional Review Board of Asan Medical Center. Written informed consent was obtained from all participants and/or their legal guardians. All methods from this study were performed in accordance with the relevant guidelines and regulations.

\section{Measurements}

\section{Usual gait speed}

In the evaluation of usual gait speed, participants were instructed to walk, from a standing point, a total of $7 \mathrm{~m}$ on a level indoor surface, with no turns, at the pace most comfortable and usual for them. Using a standard digital stopwatch, a trained tester measured the 4-m transit time between the first footstep at the starting line to the first footstep at the 4-m line. Accelerating and decelerating sections of $1.5 \mathrm{~m}$ each were excluded from the measurement. The result was defined as the gait speed. ${ }^{16}$

\section{Geriatric conditions}

Trained nurses administered a comprehensive geriatric assessment to evaluate common geriatric conditions. Multimorbidity was defined as having $\geq 5$ of the 11 physiciandiagnosed illnesses including angina, arthritis, asthma, cancer, chronic lung disease, congestive heart failure, diabetes, heart attack, hypertension, kidney disease, and stroke. A short physical performance battery (SPPB) score was calculated from three components: the ability to stand for up to $10 \mathrm{~s}$ with feet positioned in three ways (together sideby-side, semi-tandem, and tandem); the time to complete a $4-\mathrm{m}$ walk, and the time to rise from a chair five times. ${ }^{17,18}$ The resultant SPPB scores ranged from 0 to 12 , a higher score indicating a better level of function. Disability was defined as requiring assistance in performing any of the seven 
activities of daily living (ADLs) including bathing, continence, dressing, eating, toileting, transferring, and washing face and hands, or 10 instrumental activities of daily living (IADLs) including food preparation, household chores, going out a short distance, grooming, handling finances, laundry, managing own medications, shopping, transportation, and using a telephone. ${ }^{19}$ Depression was determined if the score on the Korean version of the Center for Epidemiological Studies Depression (CES-D) scale was $>20 .{ }^{20}$ Cognitive dysfunction was determined if the score on the Korean version of the Mini-Mental State Examination was $<24 .{ }^{21}$ Polypharmacy was defined as taking five or more prescription medications. ${ }^{15}$

\section{Frailty assessment}

We used a frailty index, the Cardiovascular Health Study (CHS) frailty phenotype, and the Korean version of the Fatigue, Resistance, Ambulation, Illnesses, and Loss of weight (K-FRAIL) questionnaire in this study to evaluate the associations between the usual gait speed and the frailty status.

\section{Frailty index}

For quantitative evaluation of frailty burden, we built the frailty index based on the widely accepted procedure suggested by Rockwood and colleagues..$^{22}$ The frailty index is a sensitive, quantitative predictor of adverse health outcomes, because it includes underlying diseases, physical and cognitive impairments, psychosocial risk factors, geriatric syndromes (eg, falls, delirium, and urinary incontinence), and disability. ${ }^{22}$ Of 70 previously suggested frailty index components, 34 were selected and utilized to build the present version. The specific components of our frailty index are listed in Table S1. The frailty index was calculated as the cumulative deficit of the 34 items and ranged from 0 to 1 , a higher score indicating a greater frailty burden.

\section{CHS frailty phenotype}

We evaluated the frailty phenotype according to the widely accepted CHS frailty criteria. ${ }^{5}$ These criteria, developed by Fried and coworkers, assigns 1 point to each of the five following components: 1) exhaustion ("moderate or most of the time during the past week" for either of the following statements: "I felt that everything I did was an effort" or "I could not get going"); 2) low activity (lowest quintile in physical activity level measured using the International Physical Activity Questionnaires Short Form 16); 3) slowness (usual gait speed $<0.8 \mathrm{~m} / \mathrm{s}$ in the 4 -m walk test); 4) weakness (dominant hand grip strength $<26 \mathrm{~kg}$ for men and $<17 \mathrm{~kg}$ for women); 5) weight loss (unintentional weight loss $>3 \mathrm{~kg}$ during the previous 6 months). Participants were classified as robust ( 0 points), prefrail (1-2 points), or frail (3-5 points).

\section{K-FRAIL questionnaire}

In this study, the K-FRAIL questionnaire was used as a screening measure for frailty status. ${ }^{23}$ This simple screening tool of five self-reported items has been validated in many populations, ${ }^{24-26}$ including Koreans, and it can be assessed within 2 or 3 minutes with no requirement of special equipment or training. ${ }^{6}$ It assigns 1 point to each of the following five components: 1) fatigue (response of "all of the time" or "most of the time" to the following question: "How much of the time during the past 4 weeks did you feel tired?"); 2) resistance (positive response to the question, "By yourself and not using aids, do you have any difficulty walking up 10 steps without resting?"); 3) ambulation (positive response to the question, "By yourself and not using aids, do you have any difficulty walking $300 \mathrm{~m}$ ?"); 4) illnesses (five or more self-reported physician diagnoses of hypertension, diabetes, cancer excluding a minor skin cancer, chronic lung disease, heart attack, congestive heart failure, angina, asthma, arthritis, stroke, or kidney disease); and 5) loss of weight ( $>5 \%$ in the past year). Participants were classified as robust (0 points), prefrail (1-2 points), or frail (3-5 points).

\section{Data analysis}

The independent $t$-test for continuous variables and the $\chi^{2}$ test for categorical variables were used to evaluate the participants' characteristics. Sex-specific gait-speed quartiles were calculated and used to group participants into gait-specific categories. We used linear regression analysis and Pearson correlation analysis to assess the associations between gait speed and age, frailty. The Kaplan-Meier plot, log-rank test and Cox proportional hazard model were used to determine the associations between gait-speed categories and outcomes. Additionally, receiver operating characteristic (ROC) analyses with comparisons of the area under the curve were performed to assess the predictive ability for gait speed and frailty outcomes. The statistical analyses were performed using STATA 15.0 (StataCorp, College Station, TX, USA).

\section{Results \\ Baseline characteristics and distributions of gait speed according to age and sex}

Among the 1,350 people (602 men and 748 women) examined between October 2014 and June 2017, two women were excluded for missing gait-speed data and frailty status. 


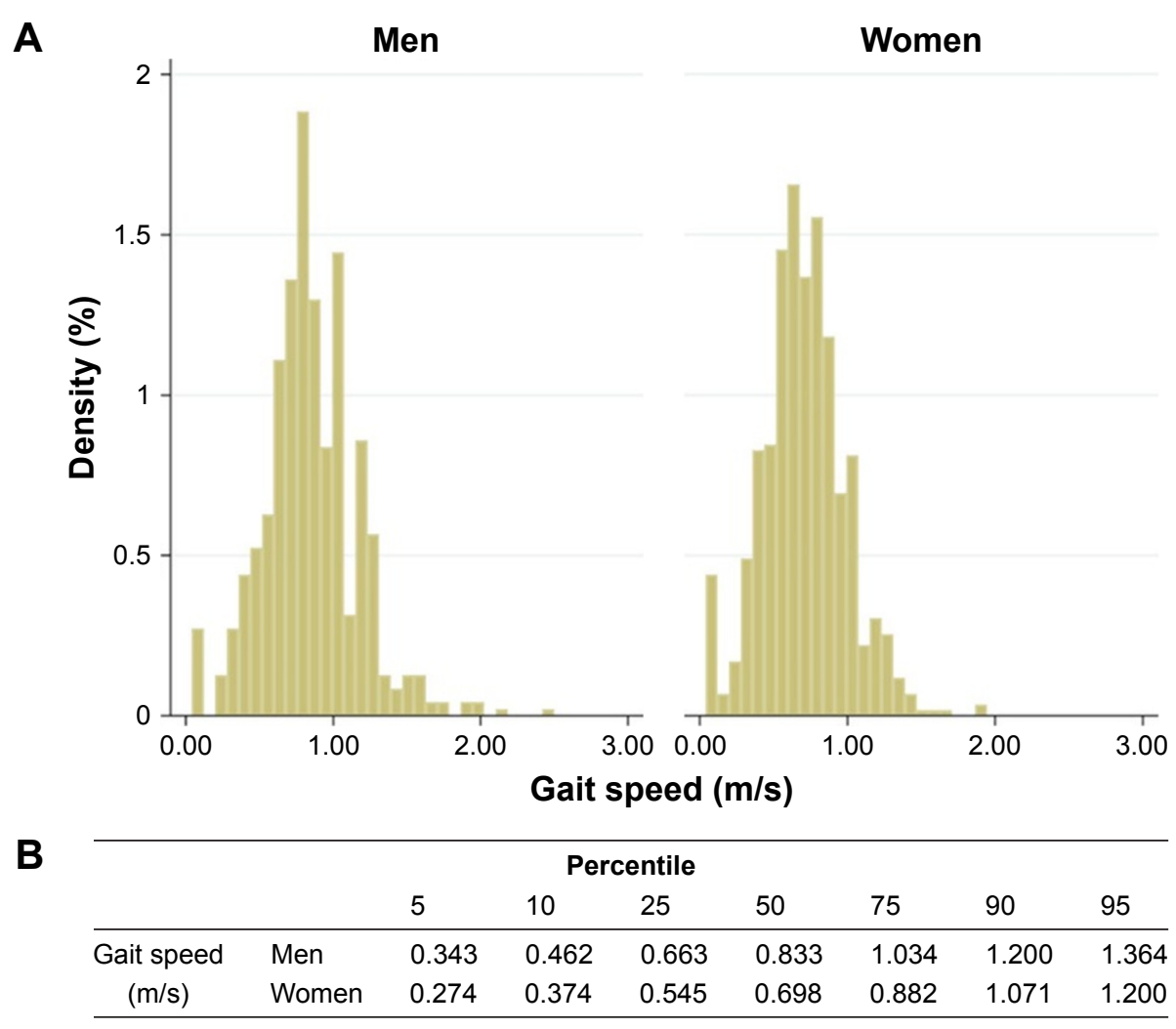

Figure I Distribution of gait speed from the Aging Study of Pyeongchang Rural Area.

Note: Distribution of gait speed by (A) sex and (B) sex-specific percentiles of gait speed.

Consequently, 1,348 (602 men and 746 women) were included in the analysis for this study. As shown in Figure 1, gait speed was slower in women (mean $0.709 \mathrm{~m} / \mathrm{s}$, standard deviation [SD] $0.283 \mathrm{~m} / \mathrm{s}$ ) than in men (mean $0.850 \mathrm{~m} / \mathrm{s}, \mathrm{SD}$ $0.321 \mathrm{~m} / \mathrm{s})$, with statistical significance $(P<0.001)$. Although the women in the study population were older than the men (mean 76.29, SD 6.67 vs mean 74.97, SD 6.04, $P<0.001$ ), the sex-dependent gait-speed difference $(0.141 \mathrm{~m} / \mathrm{s})$ could not be explained by the difference of age, by linear regression analysis showing a coefficient of $-0.017 \mathrm{~m} / \mathrm{s}$ of gait speed per 1 year increment of age. Therefore, we classified the study population into fast and slow walkers (by sex-specific median gait speed) for descriptive characteristics and used the sex-specific gait-speed quartiles for the other analyses.

When the gait speeds of participants were divided into three categories according to the known cutoff values of 0.6 and $1.0 \mathrm{~m} / \mathrm{s}, 108(17.94 \%), 310$ (51.50\%), and $184(30.56 \%)$ men had a gait speed of $<0.6,0.6-1.0$, and $\geq 1.0 \mathrm{~m} / \mathrm{s}$, respectively. ${ }^{9,10}$ On the other hand, 254 (34.05\%), 382 (51.21\%), and $110(14.75 \%)$ women had a gait speed of $<0.6,0.6-1.0$, and $\geq 1.0 \mathrm{~m} / \mathrm{s}$, respectively. With respect to the sex-specific quartiles for gait speed, 161 (26.74\%), 155 (25.75\%), 149 (24.75\%), and 137 (22.76\%) men along with 194 (26.01\%), 189 (25.34\%), 192 (25.74\%), and $171(22.92 \%)$ women were classified into the 1 st to 4 th quartiles, respectively.

As displayed in Table 1, slow walkers were older, with more multimorbidity, lower grip strength, worse SPPB score, more frailty, more depression, more cognitive dysfunction, and a fall-down history for the previous 1 year.

\section{Gait speed is associated with frailty status}

As shown in Figure 2, gait speed was associated with age and frailty index in both men and women. By linear regression analysis, age (standardized beta $[\beta]=-0.342, P<0.001$ ) was associated with gait speed after adjusting for sex. Frailty index was associated with gait speed either without adjustment $(\beta=-0.589, P<0.001)$ or with adjustment with age and $\operatorname{sex}(\beta=-0.534, P<0.001)$.

When gait speed was classified into the four categories by sex-specific quartile, the slower gait-speed categories were associated with the worse frailty status by both the K-FRAIL questionnaire (Pearson correlation coefficient $[\rho]=-0.334$, $P<0.001)$ and the CHS frailty phenotype $(\rho=-0.480$, $P<0.001)$. As shown in Figure 3, no participant in the $1 \mathrm{st}$ quartile was robust according to the CHS frailty phenotype, and $172(55.8 \%)$ in the 4th quartile were robust according to the K-FRAIL questionnaire. 
Table I Baseline characteristics compared between slow walkers (gait speed slower than sex-specific median) and fast walkers

\begin{tabular}{|c|c|c|c|c|c|}
\hline \multirow[t]{3}{*}{ Characteristics } & Slow walker & \multirow[t]{3}{*}{$\%$ or SD } & Fast walker & \multirow[t]{3}{*}{$\%$ or SD } & \multirow[t]{3}{*}{$P$-value } \\
\hline & \multirow{2}{*}{$\begin{array}{l}\mathbf{N}=68 \mathrm{I} \\
\mathbf{N} \text { or mean }\end{array}$} & & \multirow{2}{*}{$\frac{N=667}{N \text { or mean }}$} & & \\
\hline & & & & & \\
\hline Age (mean, SD) & 77.4 & 6.8 & 74.0 & 5.5 & $<0.001$ \\
\hline Female (n, \%) & 365 & 53.6 & 381 & 57.1 & 0.213 \\
\hline Education years (mean, SD) & 4.9 & 3.1 & 5.4 & 3.7 & 0.010 \\
\hline Low income $(\mathrm{n})$ & 54 & 7.9 & 35 & 5.2 & 0.061 \\
\hline Multimorbidity (n) & 310 & 45.5 & 221 & 33.1 & $<0.001$ \\
\hline $\begin{array}{l}\text { Dominant grip strength } \\
\text { (mean, SD) }\end{array}$ & 19.9 & 9.5 & 24.9 & 9.6 & $<0.001$ \\
\hline SPPB score (mean, SD) & 6.6 & 2.5 & 9.4 & 1.9 & $<0.001$ \\
\hline K-FRAIL score (mean, SD) & 1.6 & 1.2 & 0.9 & 1.1 & $<0.001$ \\
\hline CHS score (mean, SD) & 2.4 & 1.1 & 1.3 & I.I & $<0.001$ \\
\hline ADL disability (n, \%) & 125 & 18.4 & 56 & 8.4 & $<0.001$ \\
\hline IADL disability (n, \%) & 294 & 43.2 & 150 & 22.5 & $<0.001$ \\
\hline Depression (n, \%) & 102 & 15.0 & 34 & 5.1 & $<0.001$ \\
\hline Cognitive dysfunction (n, \%) & 270 & 39.6 & 125 & 18.7 & $<0.001$ \\
\hline Polypharmacy (n, \%) & 193 & 28.3 & 113 & 16.9 & $<0.001$ \\
\hline Fall history for the previous I year (mean, SD) & 0.3 & 1.2 & 0.2 & 0.6 & 0.001 \\
\hline
\end{tabular}

Abbreviations: ADL, activity of daily living; CHS, Cardiovascular Health Study; IADL, instrumental activity of daily living; K-FRAIL, the Korean version of the Fatigue, Resistance, Ambulation, Illnesses, and Loss of weight questionnaire; SD, standard deviation; SPPB, short physical performance battery.

To evaluate the associations between the subcomponents of the frailty measures and gait speed, multivariable linear regression analyses with adjustment for age and sex were performed. In the K-FRAIL questionnaire, resistance $(\beta=-0.137, P<0.001)$ and ambulation $(\beta=-0.171$, $P<0.001)$ were associated with gait speed. In the CHS frailty phenotype, exhaustion $(\beta=-0.085, P=0.001)$ and grip strength $(\beta=-0.310, P<0.001)$ were associated with gait speed when the slow gait-speed item was not included in the regression analysis.

\section{Gait speed can predict adverse outcomes as effectively as frailty status in older adults}

During the mean follow-up period of 21.5 months (SD 7.88), 23 participants (1.71\%) died and $93(6.90 \%)$ were institutionalized due to functional impairment. We considered these as the major adverse health outcomes in this study. When compared with participants without adverse outcomes, participants with outcomes were older $(80.78 \pm 7.80$ vs $75.23 \pm 6.07$ years old, $P<0.001$ by $t$-test) and had a higher baseline frailty index $(0.31 \pm 0.14$ vs $0.21 \pm 0.11, P<0.001$ by $t$-test $)$.

As shown in Figure 4, future survival without mortality or institutionalization by functional decline was affected by the sex-specific gait-speed quartile (log rank test $P<0.001$ ). Classification into the 1st quartile of sex-specific gait speed was associated with the increased risk of death or institutionalization by functional decline, either as unadjusted or as adjusted by age, sex education years, household income, multimorbidity, polypharmacy, and cognitive status in the Cox proportional hazard model (Table 2 ). Sensitivity analyses excluding participants with observation periods of $<1$ year were formed, showing similar trends of association between slower gait speed and risk of death or institutionalization by functional decline (Table S2). Secondary analysis for outcome relevance of lowest sex-specific gait-speed quartile was performed, separately for mortality and institutionalization. For mortality, gait speed of lowest sex-specific quartile was associated with increased mortality (hazard ratio [HR] 3.37, 95\% confidence interval [CI] 1.25-9.08) in the Cox proportional hazard model. However, this association was attenuated when adjusted for age, sex (HR 2.54, 95\% CI 0.88-7.28). When Cox regression analysis was performed with the outcome variable of institutionalization, gait speed of lowest sex-specific quartile was associated with institutionalization (HR 2.26, 95\% CI 1.45-3.53). The association became attenuated when adjusted with age and sex (HR 1.59, 95\% CI 0.98-2.58).

Since the frailty index has been known for its predictability of mortality and institutionalization by functional decline in older populations, we compared it in this respect with gait speed. By ROC analysis (Figure 5), the area under the curve was $0.712(95 \%$ CI $0.662-0.762)$ for the frailty index and 0.673 (95\% CI $0.619-0.728)$ for gait speed, thus indicating no statistically significant difference in outcome prediction $(P=0.059)$. 

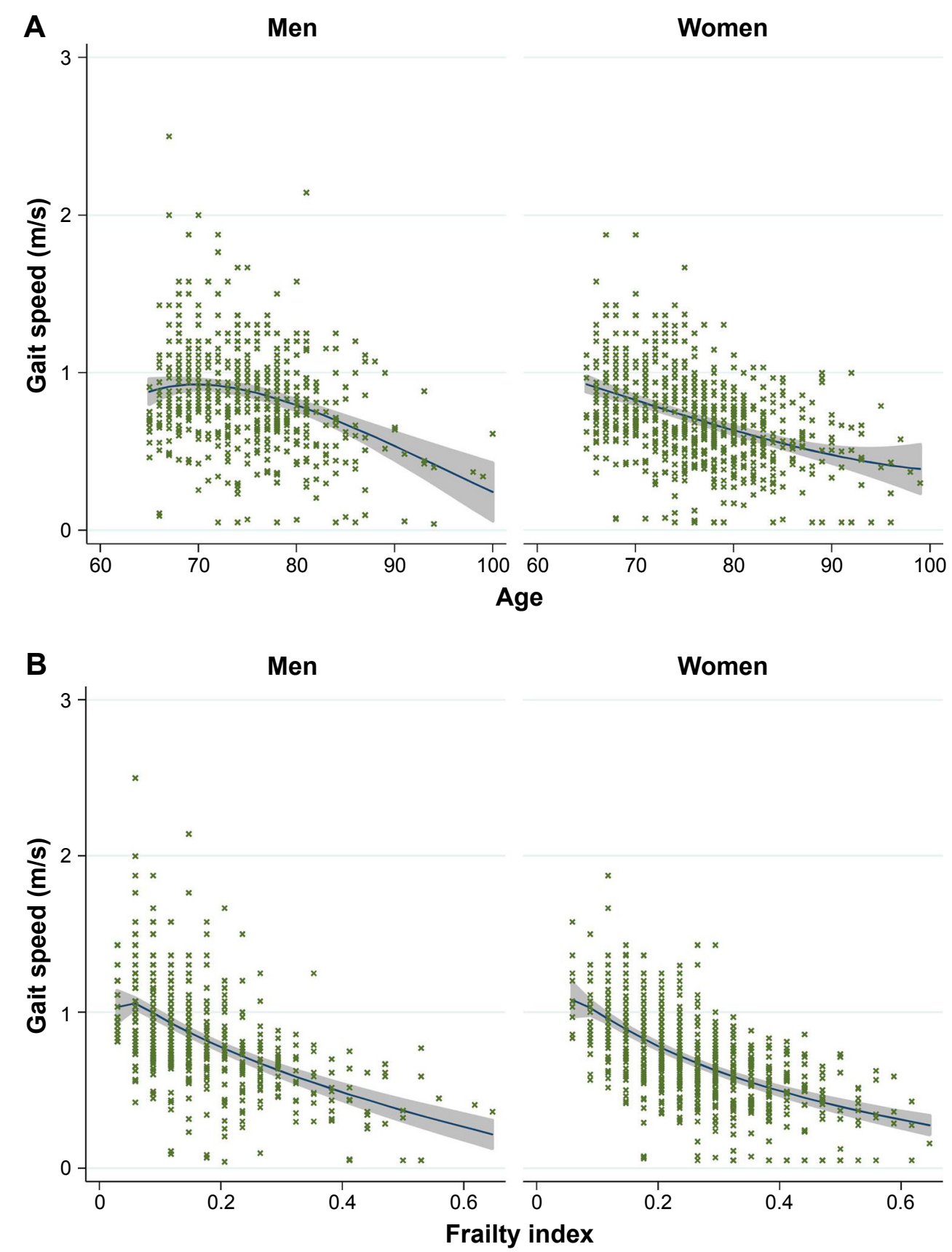

Women

$95 \% \mathrm{Cl} \longrightarrow$ Predicted line $\times$ Gait speed

Figure 2 Scatterplots showing distributions of gait speed in men and women.

Notes: $(\mathbf{A})$ by age and (B) by frailty index. The line and shading denote the predicted gait speed by fractional polynomial regression and the $95 \%$ confidence interval, respectively.

\section{Discussion}

In this study, we evaluated the distributions of gait speed according to sex, age, and frailty index among older rural community-dwelling Korean people and found that gait speed is associated with frailty status and the geriatric clinical outcome of combined mortality and institutionalization. To the best of our knowledge, this is the first study in Korea to comprehensively assess the gait speed in a longitudinal prospective cohort that can represent the general Korean rural population ${ }^{15}$ in a descriptive and analytic manner.

Although the clinical value of gait speed to assessment of physical performance, frailty status, and even neurological performance in older people has been established with cumulative evidence, especially for Western populations, ${ }^{3,9,10,27-30}$ 
A

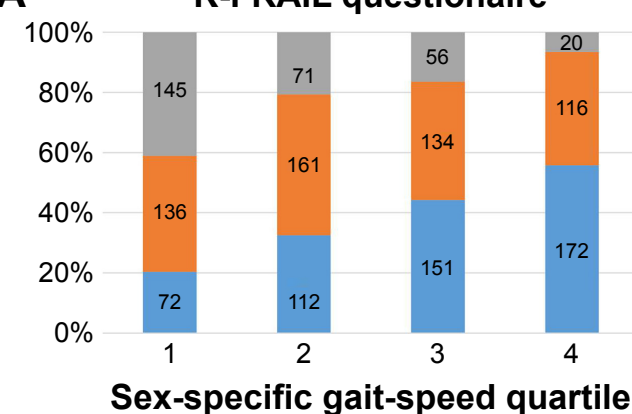

B

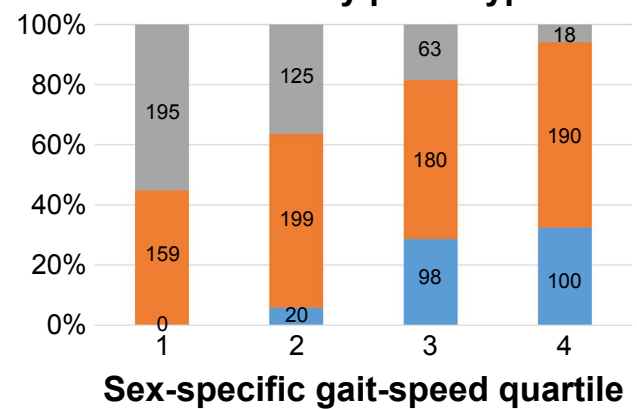

Frail $\square$ Prefrail $\square$ Robust

Figure 3 Frailty status is associated with gait-speed classification by sex-specific gait-speed quartile.

Note: The numbers in the graphs represent the absolute number of participants classified in each group for (A) the K-FRAIL questionnaire and (B) the CHS frailty phenotye.

Abbreviations: CHS, Cardiovascular Health Study; K-FRAIL, the Korean version of the Fatigue, Resistance, Ambulation, Illnesses, and Loss of weight questionnaire.

there has been insufficient data on the distributions and clinically meaningful cutoff values for gait speed among older Asian populations.

Notably, previous studies from Asian countries have suggested a higher cutoff value for slow gait speed relative to the values for Western populations. For example, a study by Tanimoto and colleagues ${ }^{31}$ suggested a cutoff value from the lowest quartile of gait speed: $<1.27 \mathrm{~m} / \mathrm{s}$ for men and $<1.29 \mathrm{~m} / \mathrm{s}$ for women. In this study, participants were recruited through local newspapers and by the local welfare commissioner, thereby leaving possibilities of bias in the study population. On the other hand, a Taiwanese group

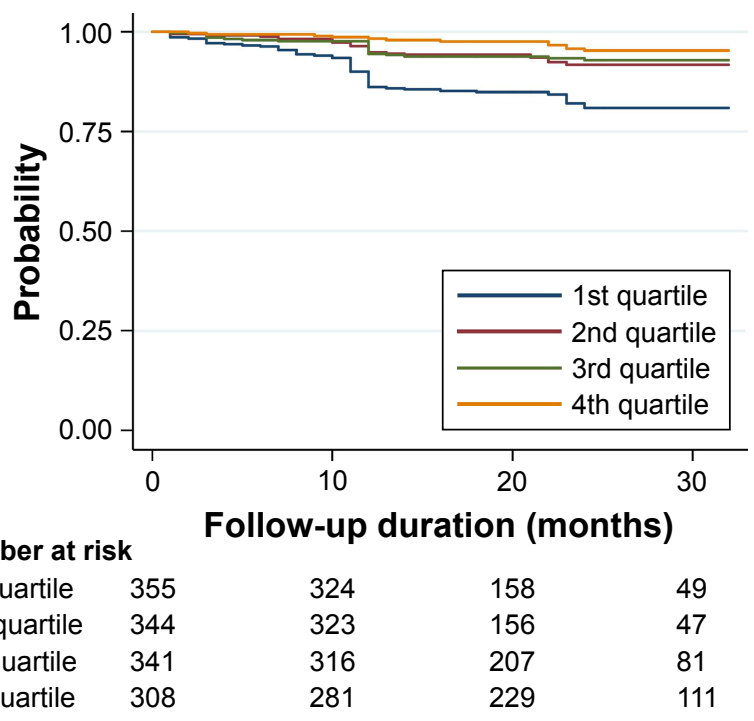

Figure 4 Kaplan-Meier curve for survival of participants without mortality or institutionalization by functional decline, with "number at risk" table.

Notes: Mean times to cutoff were 28.08, 30.47, 30.74, and 31.20 months for Ist, $2 \mathrm{nd}$, 3rd, and 4th quartiles, respectively. Total observed person-month were 9,406.64, II,090.26, 8,484.02, and 10,263.04 person-month for Ist, 2nd, 3rd, and 4 th quartiles, respectively. in the I-Lan Longitudinal Aging Study (ILAS) suggested a gait-speed cutoff value of $1 \mathrm{~m} / \mathrm{s}$ for slow gait speed ${ }^{10}$ after assessing ILAS data showing gait-speed distributions of $1.5 \pm 0.5 \mathrm{~m} / \mathrm{s}$ for men and $1.3 \pm 0.4 \mathrm{~m} / \mathrm{s}$ for women. ${ }^{32}$ For that cohort, participants were randomly sampled in the Yuanshan Township of I-Lan County in order to limit the possible bias which may oversample healthier people.

In our study, we analyzed the data of the ASPRA study, which involved a longitudinal prospective cohort in a mountainous region of PyeongChang County, Korea. Because this region is distant from major cities, the population remains relatively stable, with $<5 \%$ of older adults immigrating or

Table 2 Cox proportional hazard analysis showing the effect of gait speed on combined mortality and institutionalization during follow-up period

\begin{tabular}{|c|c|c|c|}
\hline Model & HR & $95 \% \mathrm{Cl}$ & $P$-value \\
\hline \multicolumn{4}{|l|}{ Model I } \\
\hline Ist quartile & 4.76 & $2.56-8.88$ & $<0.001$ \\
\hline 2nd quartile & 1.93 & $0.96-3.90$ & 0.066 \\
\hline 3rd quartile & 1.7 & $0.84-3.44$ & 0.139 \\
\hline \multicolumn{4}{|c|}{ 4th quartile (reference) } \\
\hline \multicolumn{4}{|c|}{ Model 2} \\
\hline Ist quartile & 2.43 & $1.24-4.76$ & 0.009 \\
\hline 2nd quartile & 1.43 & $0.68-2.90$ & 0.362 \\
\hline $3 r d$ quartile & 1.35 & $0.66-2.75$ & 0.407 \\
\hline \multicolumn{4}{|c|}{ 4th quartile (reference) } \\
\hline \multicolumn{4}{|c|}{ Model 3} \\
\hline Ist quartile & 2.31 & $1.20-4.49$ & 0.013 \\
\hline 2nd quartile & 1.26 & $0.63-2.53$ & 0.510 \\
\hline $3 r d$ quartile & 1.15 & $0.54-2.43$ & 0.720 \\
\hline 4th quartile ( & & & \\
\hline
\end{tabular}

Notes: Model I, unadjusted model; Model 2, age-, sex-adjusted model; Model 3, age, sex, education years, household income, multimorbidity, polypharmacy, and cognitive impairment-adjusted model.

Abbreviations: $\mathrm{Cl}$, confidence interval; $\mathrm{HR}$, hazard ratio. 


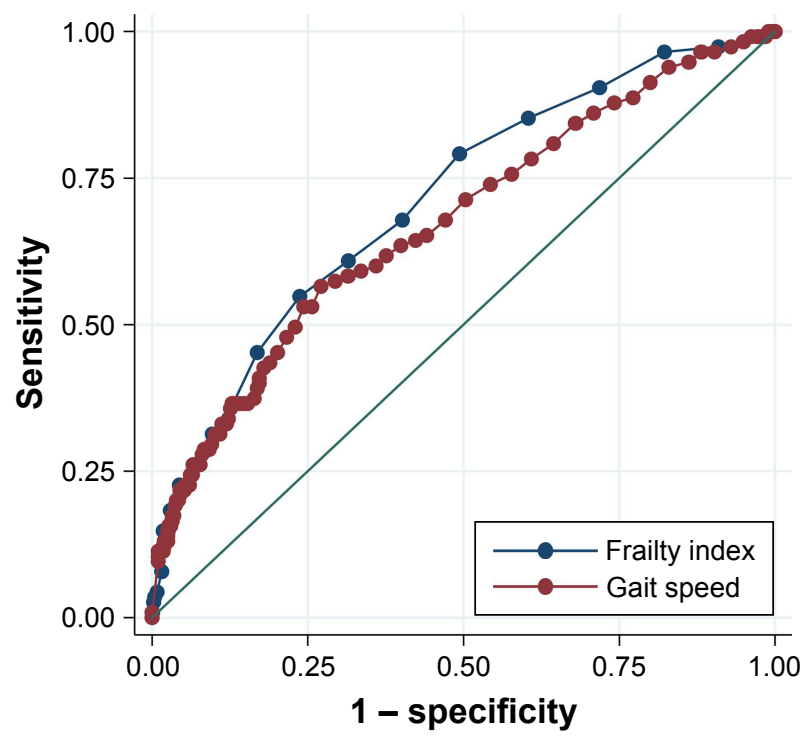

Figure $\mathbf{5}$ Receiver operating characteristic curves for the frailty index and gait speed for prediction of combined mortality and institutionalization during the follow-up period.

emigrating from the area with the exception of morbidity or mortality. Also, residents receive medical and preventive care almost exclusively from the regional Community Health Posts operated by the National Health Service. ${ }^{15}$ A comparative analysis with the sixth Korea National Health and Nutrition Examination Survey, furthermore, showed that the ASPRA population can represent the general Korean rural communitydwelling older population..$^{15}$ Therefore, the gait-speed distributions and associations with age, sex, and frailty status in our study might be considered to represent those of the general older Korean rural community-dwelling population.

The gait speeds in our study are slower than those found in previous Japanese ${ }^{31}$ and Taiwanese ${ }^{32}$ studies. This may be partially explained by the higher age in ASPRA compared with ILAS as well as the inclusion in ASPRA of even the frailest participants, who might not be included in the Japanese study.

Traditionally, gait speeds in older adults have been classified into three categories $(<0.6,0.6-1$, and $\geq 1 \mathrm{~m} / \mathrm{s})$ or two categories $(<0.8$ and $\geq 0.8 \mathrm{~m} / \mathrm{s}) .{ }^{9}, 10$ Although the AGWS adopted this cutoff value $(0.8 \mathrm{~m} / \mathrm{s})$ as its criterion for Asian sarcopenia diagnosis, it would classify nearly half of men $(272,45.18 \%)$ and too many women $(483,64.75 \%)$ as slow walkers in our study population. Even when the $0.6 \mathrm{~m} / \mathrm{s}$ cutoff was used, 254 (34.05\%) women were classified as slow walkers, because the sex-dependent difference in gait speed was substantial in the ASPRA population.

Alternatively, defining low gait speed according to the sex-specific lowest quartile might be beneficial, specifically to prevent misclassification and also to meet the original goal of setting a cutoff value of impairment for a designated population..$^{33}$ Also, the sex-specific lowest quartile of gait speed was associated in this study with future adverse outcomes including death and institutionalization by functional impairment. Therefore, we suggest that the cutoff value for slow gait speed in the Korean population be $0.663 \mathrm{~m} / \mathrm{s}$ for men and $0.545 \mathrm{~m} / \mathrm{s}$ for women, which are the sex-specific 25 th percentile values of gait speed.

Considering the as-yet unmet need of physical parameter reference values for longitudinal outcome measures that can be incorporated into future Asian sarcopenia guidelines for older people, the results obtained in this study might prove helpful. However, there are several limitations to the present study. First of all, the data reflect only a rural population of older Korean people, who are more frail and of lower socioeconomic status than urban-dwelling populations. ${ }^{34}$ Also, the ASPRA focused on items included in a comprehensive geriatric assessment and diagnosis of sarcopenia and frailty; it lacks biomarker assessments and detailed physical assessments, such as gait analysis, that typically are performed in rehabilitation laboratories. In the present study, the follow-up duration for outcome assessment was short, and only a small set of participants died during the observation period. This prevented a detailed analysis of outcome relevance of sexspecific gait speed quartile on separate outcome of mortality and institutionalization. Therefore, further studies including urban populations with specific biological and physical markers, with longer observation periods, are warranted.

\section{Conclusion}

Gait speed was associated with age and frailty status and was slower in women than in men among Korean rural community-dwelling older adults. Since the sex-specific difference in gait speed was substantial, we recommend that the cutoff value for slow gait speed in older Korean adults be based on sex-specific quartiles to minimize misclassification in operationalization of sarcopenia and determination of frailty status.

\section{Acknowledgments}

We are indebted to the public health professionals and nurses of PyeongChang County Hospital, Public Health Center, and Community Health Posts for their administrative support and help in enrollment, retention, and measurements. The Aging Study of PyeongChang Rural Area was supported by PyeongChang County Hospital, PyeongChang County, 
Gangwon Province, Korea. The present study was supported, in part, by Paul Park and Maeil Dairies Co., Ltd. from the Asan Institute for Life Sciences and Corporate Relations of the Asan Medical Center, Seoul, Korea. The public health professionals and nurses of PyeongChang County Hospital were involved in data collection, but they did not have any role in the study design, analysis, or interpretation of data, in the writing of the report or in the decision to submit it for publication. Paul Park and Maeil Dairies Co., Ltd. did not have any role in the study design, in the collection, analysis, or interpretation of data; in the writing of the report; or in the decision to submit it for publication.

\section{Author contributions}

All authors participated in designing the study conception; performing the statistical analysis, data analysis, and interpretation; and drafting the manuscript. The agreement is to be accountable for all aspects of the work in ensuring that the accuracy or integrity of any part of the work is appropriately investigated and resolved. All authors read and revised this manuscript. This manuscript is the final approval of version from all authors. All authors have reviewed and agreed to be responsible for the process, accuracy, and integrity of all parts of this manuscript.

\section{Disclosure}

The authors report no conflicts of interest in this work.

\section{References}

1. Fritz S, Lusardi M. White paper: "walking speed: the sixth vital sign". $J$ Geriatr Phys Ther. 2009;32(2):46-49.

2. Middleton A, Fritz SL, Lusardi M. Walking speed: the functional vital sign. J Aging Phys Act. 2015;23(2):314-322.

3. Studenski S, Perera S, Patel K, et al. Gait speed and survival in older adults. JAMA. 2011;305(1):50-58.

4. Clegg A, Young J, Iliffe S, Rikkert MO, Rockwood K. Frailty in elderly people. Lancet. 2013;381(9868):752-762.

5. Fried LP, Tangen CM, Walston J, et al. Frailty in older adults: evidence for a phenotype. J Gerontol A Biol Sci Med Sci. 2001;56(3):M146-M156.

6. Jung HW, Yoo HJ, Park SY, et al. The Korean version of the FRAIL scale: clinical feasibility and validity of assessing the frailty status of Korean elderly. Korean J Intern Med. 2016;31(3):594-600.

7. Alfredsson J, Stebbins A, Brennan JM, et al. Gait speed predicts 30-day mortality after transcatheter aortic valve replacement: results from the Society of Thoracic Surgeons/American College of Cardiology transcatheter valve therapy registry. Circulation. 2016;133(14): 1351-1359.

8. Afilalo J, Eisenberg MJ, Morin JF, et al. Gait speed as an incremental predictor of mortality and major morbidity in elderly patients undergoing cardiac surgery. J Am Coll Cardiol. 2010;56(20):1668-1676.

9. Cruz-Jentoft AJ, Baeyens JP, Bauer JM, et al. Sarcopenia: European consensus on definition and diagnosis: report of the European Working Group on Sarcopenia in Older People. Age Ageing. 2010;39(4): 412-423.
10. Chen LK, Liu LK, Woo J, et al. Sarcopenia in Asia: consensus report of the Asian Working Group for Sarcopenia. J Am Med Dir Assoc. 2014; 15(2):95-101.

11. Cesari M, Kritchevsky SB, Newman AB, et al. Added value of physical performance measures in predicting adverse health-related events: results from the Health, Aging and Body Composition Study. $J$ Am Geriatr Soc. 2009;57(2):251-259.

12. Lauretani F, Russo CR, Bandinelli S, et al. Age-associated changes in skeletal muscles and their effect on mobility: an operational diagnosis of sarcopenia. J Appl Physiol (1985). 2003;95(5):1851-1860.

13. Kontis V, Bennett JE, Mathers CD, Li G, Foreman K, Ezzati M. Future life expectancy in 35 industrialised countries: projections with a Bayesian model ensemble. Lancet. 2017;389(10076):1323-1335.

14. Jung HW, Kim SW, Ahn S, et al. Prevalence and outcomes of frailty in Korean elderly population: comparisons of a multidimensional frailty index with two phenotype models. PLoS One. 2014;9(2):e87958.

15. Jung HW, Jang IY, Lee YS, et al. Prevalence of frailty and aging-related health conditions in older Koreans in rural communities: a crosssectional analysis of the Aging Study of Pyeongchang Rural Area. $J$ Korean Med Sci. 2016;31(3):345-352.

16. Jang IY, Lee CK, Jung HW, et al. Urologic symptoms and burden of frailty and geriatric conditions in older men: the Aging Study of PyeongChang Rural Area. Clin Interv Aging. 2018;13:297-304.

17. Guralnik JM, Simonsick EM, Ferrucci L, et al. A short physical performance battery assessing lower extremity function: association with self-reported disability and prediction of mortality and nursing home admission. J Gerontol. 1994;49(2):M85-M94.

18. Pavasini R, Guralnik J, Brown JC, et al. Short Physical Performance Battery and all-cause mortality: systematic review and meta-analysis. BMC Med. 2016;14(1):215.

19. Won CW, Yang KY, Rho YG, et al. The Development of Korean Activities of Daily Living (K-ADL) and Korean Instrumental Activities of Daily Living (K-IADL) Scale. J Korean Geriatr Soc. 2002;6(2): $107-120$.

20. Radloff LS. The CES-D scale a self-report depression scale for research in the general population. Appl Psychol Meas. 1977;1(3):385-401.

21. Kang Y, Na DL, Hahn S. A validity study on the Korean Mini-Mental State Examination (K-MMSE) in dementia patients. J Korean Neurol Assoc. 1997;15(2):300-308.

22. Rockwood K, Andrew M, Mitnitski A. A comparison of two approaches to measuring frailty in elderly people. J Gerontol A Biol Sci Med Sci. 2007;62(7):738-743.

23. Morley JE, Malmstrom TK, Miller DK. A simple frailty questionnaire (FRAIL) predicts outcomes in middle aged African Americans. J Nutr Health Aging. 2012;16(7):601-608.

24. Woo J, Yu R, Wong M, Yeung F, Wong M, Lum C. Frailty screening in the community using the FRAIL scale. J Am Med Dir Assoc. 2015;16(5):412-419.

25. Gleason LJ, Benton EA, Alvarez-Nebreda ML, Weaver MJ, Harris MB, Javedan H. FRAIL questionnaire screening tool and short-term outcomes in geriatric fracture patients. J Am Med Dir Assoc. 2017;18(12): 1082-1086.

26. Malmstrom TK, Miller DK, Morley JE. A comparison of four frailty models. J Am Geriatr Soc. 2014;62(4):721-726.

27. Studenski S, Perera S, Wallace D, et al. Physical performance measures in the clinical setting. J Am Geriatr Soc. 2003;51(3):314-322.

28. Rosano C, Brach J, Studenski S, Longstreth WT Jr, Newman AB. Gait variability is associated with subclinical brain vascular abnormalities in high-functioning older adults. Neuroepidemiology. 2007; 29(3-4):193-200.

29. Rosano C, Studenski SA, Aizenstein HJ, Boudreau RM, Longstreth WT Jr, Newman AB. Slower gait, slower information processing and smaller prefrontal area in older adults. Age Ageing. 2012;41(1):58-64.

30. Beavers KM, Beavers DP, Houston DK, et al. Associations between body composition and gait-speed decline: results from the health, aging, and body composition study. Am J Clin Nutr. 2013;97(3):552-560. 
31. Tanimoto $Y$, Watanabe M, Sun W, et al. Association between sarcopenia and higher-level functional capacity in daily living in communitydwelling elderly subjects in Japan. Arch Gerontol Geriatr. 2012; 55(2):e9-e13.

32. Liu LK, Lee WJ, Liu CL, et al. Age-related skeletal muscle mass loss and physical performance in Taiwan: implications to diagnostic strategy of sarcopenia in Asia. Geriatr Gerontol Int. 2013;13(4):964-971.
33. Searle SD, Mitnitski A, Gahbauer EA, Gill TM, Rockwood K. A standard procedure for creating a frailty index. BMC Geriatr. 2008;8:24.

34. Jang IY, Jung HW, Lee CK, et al. Rural and urban disparities in frailty and aging-related health conditions in Korea. J Am Geriatr Soc. 2016; 64(4):908-911. 


\section{Supplementary materials}

Table SI The 34 components of the frailty index

- Changes in everyday activities

- Problems in getting dressed

- Problems in bathing

- Problems in carrying out personal grooming

- Urinary incontinence

- Toileting problems

- Gastrointestinal problems

- Problems in cooking

- Problems in going out alone

- Impaired mobility

- Musculoskeletal problems

- Bradykinesia of the limbs

- Poor muscle tone in limbs

- Poor limb coordination

- Poor trunk coordination

- Poor standing posture

- Irregular gait pattern
- Falls

- Mood problems

- Tiredness all the time

- Depression

- Memory changes

- Changes in general mental functioning

- History relevant to cognitive impairment or loss

- History of stroke

- History of diabetes mellitus

- Arterial hypertension

- Cardiac problems

- Myocardial infarction

- Congestive heart failure

- Lung problems

- Respiratory problems

- Malignant disease

- Other medical history
Table S2 Cox proportional hazard analysis showing the effect of gait speed on combined mortality and institutionalization during the follow-up period, excluding participants with observation periods of $<$ I year

\begin{tabular}{|c|c|c|c|}
\hline Model & HR & $95 \% \mathrm{Cl}$ & $P$-value \\
\hline \multicolumn{4}{|l|}{ Model I } \\
\hline Ist quartile & 4.87 & $2.25-10.53$ & $<0.001$ \\
\hline 2nd quartile & 1.89 & $0.80-4.47$ & 0.147 \\
\hline 3rd quartile & 1.51 & $0.60-3.83$ & 0.384 \\
\hline \multicolumn{4}{|c|}{ 4th quartile (reference) } \\
\hline \multicolumn{4}{|c|}{ Model 2} \\
\hline Ist quartile & 2.69 & $1.18-6.11$ & 0.018 \\
\hline 2nd quartile & 1.38 & $0.58-3.30$ & 0.466 \\
\hline $3 r d$ quartile & 1.26 & $0.50-3.21$ & 0.623 \\
\hline \multicolumn{4}{|c|}{ 4th quartile (reference) } \\
\hline \multicolumn{4}{|c|}{ Model 3} \\
\hline Ist quartile & 2.51 & $1.08-5.84$ & 0.032 \\
\hline 2nd quartile & 1.34 & $0.55-3.24$ & 0.517 \\
\hline 3rd quartile & 1.25 & $0.49-3.18$ & 0.646 \\
\hline 4th quartile ( & & & \\
\hline
\end{tabular}

Notes: Model I, unadjusted model; Model 2, age-, sex-adjusted model; Model 3, age, sex, education years, household income, multimorbidity, polypharmacy, and cognitive impairment-adjusted model.

Abbreviations: $\mathrm{Cl}$, confidence interval; $\mathrm{HR}$, hazard ratio.
Clinical Interventions in Aging

\section{Publish your work in this journal}

Clinical Interventions in Aging is an international, peer-reviewed journal focusing on evidence-based reports on the value or lack thereof of treatments intended to prevent or delay the onset of maladaptive correlates of aging in human beings. This journal is indexed on PubMed Central, MedLine,
CAS, Scopus and the Elsevier Bibliographic databases. The manuscript management system is completely online and includes a very quick and fair peer-review system, which is all easy to use. Visit http://www.dovepress. com/testimonials.php to read real quotes from published authors. 